\title{
Application of Local and Global Eigenface Decompositions for Vehicular Detection during the COVID-19 Health Pandemic: Preliminary Results
}

\author{
Nicholas V. Scott ${ }^{1}$ and Linnette M. Martinez ${ }^{2}$ \\ ${ }^{1}$ Riverside Research, Open Innovation Center, Dayton Research Center \\ 2640 Hibiscus Way, Beavercreek, Ohio, 45431, USA \\ nscott@riversideresearch.org; 1m4432@nyu.edu \\ ${ }^{2}$ New York University, Tandon School of Engineering, Polytechnic Institute \\ 6 MetroTech Center, Brooklyn, NY, 11201, USA
}

\section{Extended Abstract}

The current health pandemic has precipitated a significant change in vehicular traffic due to quarantine procedures enacted by local and state governments. Such changes have a significant effect on transportation affecting such issues as road maintenance and traffic fluidity which impact the movement of vital resources throughout the state. With predicted increases in the modulation of traffic flow into the future, especially in esoteric hard-to-reach areas, there is a need for inexpensive, innovative, and effective ways to estimate the mean number of vehicles passing on the road per unit time. To address this desire, off-the-shelf panchromatic camera technology and global and local eigenface decompositions based on manifold learning are exploited to estimate the number of cars passing per unit time on a semi-busy road. The premise of the approach is that an ensemble of decompositions could possibly succeed in robust vehicular detection where single algorithms fail. Eigenface methods represent a different form of vehicular detection with roots in traditional facial recognition [1] as opposed to more complicated methods such as mask-based, deep learning algorithms which can be expensive.

A Brinno TLC300PRO RGB camera, mounted on a small tripod directed outside a window in a residence was configured to capture AVI files of traffic flow on a road in Beavercreek, Ohio, USA from April 21, 2020 to May 12, 2020. Images sampled at a frequency of 2 images per minute were extracted over a 3-hour interval on the first day in the morning. Fiftyone training image chips with the dimensions of 21 x 76 pixels, were extracted, vectorized, and placed in a 1596 x 51 element matrix. This data was used to derive global and local eigenfaces for the imagery possessing no vehicles. Thirty-seven test image chips containing imagery with and without vehicles and possessing the same dimensions, were extracted, vectorized, and placed into a similar matrix. This test data was subsequently projected into three different eigenspaces corresponding to three decompositions - principal component analysis (PCA) [2], Laplacian eigenmap (LE) analysis [3], and nonnegative matrix factorization (NMF) decomposition [4], to test for the presence of vehicles.

Training data-based PCA eigenfaces were used to perform eigenface projection of imagery where weight abundances appear as an embedded manifold. A two-dimensional eigenspace, based on the projection of the original 51 images and 37 test images possessing both vehicles and no vehicles and which used the $1^{\text {st }}$ and $2^{\text {nd }}$ eigenfaces, did produce the appropriate segmentation of imagery. Weight abundances in a two-dimensional Laplacian Eigenmap eigenspace using the $2^{\text {nd }}$ and $3^{\text {rd }}$ eigenfaces, and weight abundances in a two-dimensional nonnegative matrix factorization eigenspace using the $3^{\text {rd }}$ and the $4^{\text {th }}$ eigenfaces both comprise embedded manifolds which failed to produce strong vehicle - no vehicle data segmentation. The lack of data segmentation in two of the eigenface decomposition analyses suggests the presence of contorted manifolds. Higher dimensional information embodied in the remaining eigenfaces is needed to perform vehicular anomaly detection using the matrix transformations.

Mean weight vectors obtained from averaging over the full number of eigenfaces associated with PCA, LE analysis, and NMF analysis for the training imagery were created for vehicular anomaly detection. Projections of test imagery using the full sets of eigenfaces provided weight value sets whose distances from the mean weight vectors were calculated. Such distances provided a metric for assessing anomalous imagery data points. Each weight-based distance curve for PCA, LE, and NMF analysis provided semi-smooth trends with sporadic low values which were mixtures of true anomalous data points, designating imagery with vehicles, as well as false positives. The false positives, which caused very large root mean square 
error values for all eigenfaces, were due to sporadic camera image setting changes caused by ambient sky lighting changes. Such fluctuations caused confusion in the eigenface decompositions even with image detrending. The weight-based distance curves however do suggest that deviation from the mean nonlinear trend could be a metric for vehicular detection.

With additional post image filtration analysis to remove false positives, robust and fast vehicular anomaly detection is possible using the PCA, LE, and NMF eigenface methods. Because each algorithm represents a weak detector, use of them as an ensemble of detectors via machine learning boosting [5] is a possible method for building a robust vehicular detector. Continued research is aimed at 1) amalgamating the decompositions for ensemble learning and 2) using alternative neural network approaches, both serving as quantification of statistical temporal vehicular flow over weekly time scales.

\section{References}

[1] M. Turk, and A. Pentland, "Eigenfaces for Recognition", Journal of Cognitive Neuroscience, vol. 3, no. 1, pp. 71-86, 1991.

[2] W. M. Emery, and R. E. Thomson, Data Analysis in Physical Oceanography. New York, USA: Pergamon Press, 1997.

[3] J. A. Lee, and M. Verleysen, Nonlinear Dimensionality Reduction. New York, USA: Springer, 2007.

[4] A. Cichocki, R. Zdnunek, A. H. Phan, and S-I Amari, Nonnegative Matrix Factorization: Applications to Exploratory Multiway Data Analysis and Blind Source Separation. West Sussex, UK: John Wiley and Sons, 2009.

[5] W. L. Martinez, and A. R. Martinez, Computational Statistics Handbook with Matlab. London, UK: Chapman and Hall/CRC, 2001. 